\section{Efecto de la hidroxicloroquina sobre la sensibilidad a la insulina y el perfil de lípidos en pacientes con artritis reumatoide}

\author{
RODOLFO ARAIZA-CASILLAS ${ }^{1,2, a}$, RAÚL DÍAZ-MOLINA ${ }^{2, a, b}$, \\ MANUEL GONZÁLEZ-ORTIZ ${ }^{3, \mathrm{a}, \mathrm{b}}$, OCTAVIO MANUEL ROBINSON-NAVARRO ${ }^{2, \mathrm{a}, \mathrm{b}}$
}

\section{Effects of hydroxychloroquine on insulin sensitivity and lipid profile in patients with rheumatoid arthritis}

Background: Rheumatoid arthritis $(R A)$ is associated with a decrease in insulin sensitivity (IS), which has been identified as an independent risk factor for the development of early atherosclerosis. Hydroxychloroquine (HCQ) may have beneficial effects on glucose homeostasis and lipid profile. Aim: To assess the effect of HCQ on IS and lipid profile in patients with RA. Material and Methods: An open clinical trial was performed in 15 patients aged between 35 and 56 years. During three months, patients received $400 \mathrm{mg}$ /day of HCQ orally. Before and after the pharmacological intervention, demographic and anthropometric variables, serum glucose, total cholesterol (TC), triglycerides (TG), HDL-cholesterol, LDL-cholesterol, VLDL-cholesterol, insulin and uric acid were measured. IS was estimated as the rate of glucose clearance per minute obtained with the insulin tolerance test (KITT). Results: Baseline and final KITT values were $4.3 \pm 1.2$ and $4.80 \pm 1.1 \% / \mathrm{min}$, respectively $(p=0.03)$. Significant reductions in serum TC $(p=0.04)$ and TG $(p=0.01)$ were also observed. No other significant differences were observed. Conclusions: Oral administration of $400 \mathrm{mg}$ /day of HCQ during three months in RA patients is associated with an improvement in IS, TC and TG.

(Rev Med Chile 2013; 141: 1019-1025).

Key words: Arthritis, rheumatoid; Hydroxychloroquine; Insulin resistance.

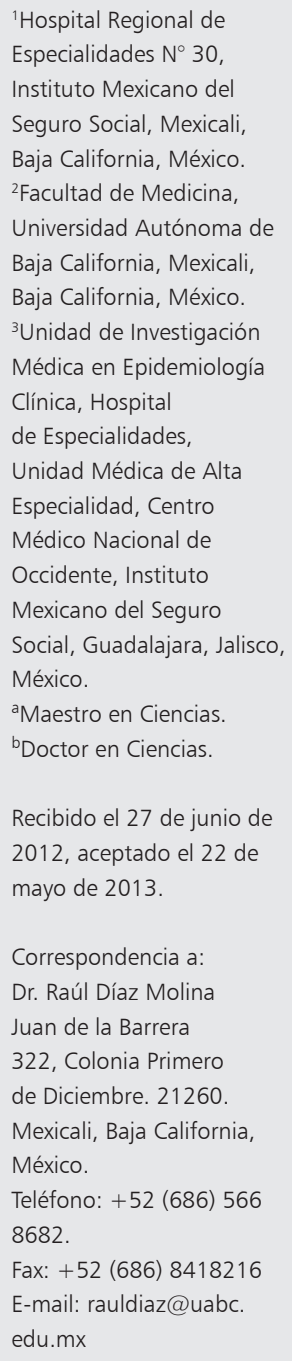

L a artritis reumatoide (AR) es la enfermedad reumática autoinmune más frecuente. La prevalencia en la población general en la mayoría de los países es de 0,5-1\%. Esta enfermedad se caracteriza por inflamación crónica generalizada que afecta principalmente a las articulaciones sinoviales y tres veces más a mujeres que a varones. La manifestación clínica principal es una poliartritis persistente con una distribución simétrica que afecta las articulaciones periféricas, en etapas avanzadas resulta en deformidades de las mismas, y en 20\% de los casos afecta sitios extraarticulares. En la mayoría de los casos la enfermedad inicia en la etapa productiva de la vida y puede producir una discapacidad física importante, teniendo un alto impacto social y económico en la población ${ }^{1}$.

No obstante el avance en el conocimiento de la AR y el desarrollo de mejores estrategias terapéuticas, los estudios observacionales muestran que esta enfermedad reduce la sobrevida. Los eventos cardiovasculares (ECV) son una causa de muerte que explica claramente el aumento en la mortalidad temprana. Recientemente se ha descrito que cerca de $50 \%$ de los pacientes con AR cursan con disminución de la sensibilidad a la insulina (SI) ligada a inflamación crónica, alteración metabó- 
lica que ha emergido como un factor de riesgo independiente para el desarrollo de aterosclerosis temprana ${ }^{2-4}$. Los pacientes con AR tienen 3,9 veces más riesgo de $\mathrm{ECV}$ comparados con la población general. Aunque no se conocen del todo los mecanismos por los cuales una disminución en la SI predispone a aterosclerosis temprana, se ha invocado la disfunción endotelial como el paso inicial en el desarrollo de esta complicación ${ }^{4-6}$.

La sensibilidad a la insulina (SI) se define como la capacidad que tienen las células del organismo de captar la glucosa plasmática inducida por la acción de la insulina ${ }^{7}$. Normalmente hay un equilibrio entre la cantidad de insulina secretada en proporción con la cantidad de glucosa que debe ser ingresada a las células. La resistencia a la insulina se presenta cuando se pierde este equilibrio, de tal manera que se necesita una mayor cantidad de insulina para poder ingresar la misma cantidad de glucosa a las células, mientras más pronunciado sea este desequilibrio, menor es la SI.

La hidroxicloroquina (HCQ) es un derivado sintético de las 4-aminoquinolonas que pertenece al grupo de los fármacos antimaláricos. Es bien tolerado y con un excelente perfil de seguridad cuando se compara con otros fármacos antiartríticos. Los efectos adversos más frecuentes son dependientes de la dosis, particularmente cuando ésta es mayor a los $400 \mathrm{mg}$ al día o cuando se administra por tiempo muy prolongado. Estos efectos se pueden presentar a nivel gastrointestinal (10-20\%), cutáneo (3\%) y ocular (1\%), los cuales son transitorios y de una magnitud leve que rara vez implica suspender el tratamiento ${ }^{8-10}$.

La HCQ, además de sus propiedades antiinflamatorias e inmunorreguladoras, muestra efectos benéficos en el metabolismo de la glucosa ${ }^{11,12}$, control glucémico ${ }^{13}$ y la disminución del riesgo de DM2 ${ }^{14}$; así como en el metabolismo de los lípidos, a través de una disminución del colesterol total (CT) y los triglicéridos (TG) plasmáticos, y un incremento en los niveles sanguíneos de HDLcolesterol $^{15,16}$. Sin embargo, en estos estudios, los cuales se han conducido tanto en modelos animales como en humanos, se han utilizado dosis de HCQ superiores a las utilizadas en la práctica clínica.

Es claro que existe una interrelación entre AR como enfermedad inflamatoria crónica y la disminución de la SI, alteraciones que parecen tener un efecto aditivo para el desarrollo de aterosclerosis en esta enfermedad. En este contexto, el papel que la HCQ puede jugar como arma terapéutica no se conoce. El objetivo de la presente investigación fue evaluar el efecto de la HCQ sobre la SI y el perfil de lípidos a dosis terapéuticas usadas en el tratamiento de pacientes con AR (400 mg diarios por vía oral), situación que no ha sido estudiada.

\section{Materiales y Métodos}

Se trató de un ensayo clínico, cuasiexperimental, con intervención farmacológica en un sólo grupo antes-después. Se incluyeron en el estudio pacientes de género indistinto entre 18 y 70 años de edad con diagnóstico de AR según los criterios del Colegio Americano de Reumatología ${ }^{17}$, tratados con una dosis estable de metotrexate de $\geq 10$ $y \leq 20 \mathrm{mg}$ por semana vía oral durante los 6 meses previos a la visita de inicio de la intervención; y en remisión de su enfermedad, la cual se estableció con base en el índice de actividad de la enfermedad para 28 articulaciones (DAS28) menor a 2,6 ${ }^{18}$. Se excluyeron los pacientes que durante los últimos 6 meses fueron tratados con fármacos modificadores de la enfermedad distintos al metotrexate, así como todos aquellos que durante el último mes se encontraban tratados con prednisona a una dosis mayor de $10 \mathrm{mg}$ por día, indometacina, inhibidores de la enzima convertidora de angiotensina (ECA), antagonistas de los receptores de la angiotensina II (ARA-II), metformina, acarbosa, $\beta$-bloqueadores, hipolipemiantes, propiltiouracilo, simpatolíticos, aspirina a dosis mayores a $1 \mathrm{~g}$ por día, o compuestos con estrógenos y progestágenos. Para controlar las diferencias entre los sujetos de estudio, éstos fueron sus propios controles y seleccionados por conveniencia.

El diagnóstico de disminución de la SI se estableció de acuerdo con el National Cholesterol Education Program-Adult Treatment Panel III (NCEP-ATP III $)^{19}$. Para descartar diabetes mellitus tipo 2 (DM2) se realizó la prueba oral de tolerancia a la glucosa $(\mathrm{OTG})^{20}$.

Todos los pacientes que ingresaron al estudio fueron tratados con $400 \mathrm{mg}$ diarios de HCQ vía oral, durante 3 meses. Antes y después de la intervención se realizó un interrogatorio y examen físico, se tomaron medidas antropométricas y signos vitales, se cuantificaron los niveles séricos 
de CT, TG, HDL-C, LDL-colesterol (LDL-C), VLDL-colesterol (VLDL-C), glucosa, insulina y ácido úrico. La SI se estimó a través de la tasa de depuración de glucosa obtenida mediante la prueba de tolerancia a la insulina (KITT) ${ }^{21-23}$.

La cuantificación de las concentraciones séricas de glucosa, TG, CT, HDL-C y ácido úrico se realizó en un analizador de química sanguínea Elan ATAC 8000 (Elan Diagnostics, Smithfield, RI, USA), siguiendo las indicaciones del fabricante. El VLDL-C se calculó como la concentración sérica de triglicéridos/5, mientras que el LDL-C se estimó mediante la fórmula de Friedewald ( $\mathrm{LDL}-\mathrm{C}=\mathrm{CT}$ - HDL-C - VLDL-C). La insulina se cuantificó a través de un ensayo inmunoenzimométrico disponible comercialmente (Monobind Inc., Lake Forest, CA, USA), de acuerdo con las instrucciones del fabricante.

El tamaño de la muestra se calculó con la fórmula para variables continuas para contrastar una prueba de hipótesis entre dos medias en un estudio antes-después ${ }^{24}$, con un nivel de confianza de $95 \%$ y un poder de $80 \%$. Se tomó como referencia un valor de desviación estándar para el valor de KITT de $0,8 \% / \mathrm{min}$, a partir de un estudio previo con diseño similar al presente ${ }^{25}$.

Se utilizó estadística descriptiva para analizar los datos demográficos y antropométricos con medidas de tendencia central y de dispersión. Para el análisis inferencial, primero se determinó la normalidad de la distribución de los datos de cada una de las variables con la prueba de Shapiro, y posteriormente se realizó una prueba de hipótesis para dos medias de grupos pareados antes-después. Se estableció un nivel de significancia estadística con un valor de $\mathrm{p}<0,05$, con un nivel de confianza de $95 \%$ y poder estadístico de la prueba de $80 \%$.

El estudio se desarrolló en apego a la Ley General de Salud en Materia de la Investigación para la Salud, así como a la Declaración de Helsinki, en su última modificación en Escocia en 2008. El estudio fue aprobado por el Comité Local de Investigación en Salud del Instituto Mexicano del Seguro Social. Se diseñó un formato para la obtención del consentimiento informado, el cual fue firmado por todos los pacientes participantes en el proyecto. Los pacientes no recibieron compensación alguna por su participación en el estudio y fueron informados oportunamente de los procedimientos y posibles inconvenientes del estudio.

\section{Resultados}

Se seleccionó una muestra de 15 pacientes cuya edad promedio fue de 46,9 \pm 11 años, con una evolución promedio de la enfermedad de $8,5 \pm 5,8$ años, un perímetro promedio de cintura de $91,7 \pm$ $4,3 \mathrm{~cm}$ y un IMC promedio de $30,9 \pm 3,3 \mathrm{~kg} / \mathrm{m}^{2}$. Este último dato refleja el grado de obesidad de los pacientes. Ninguno presentó datos de DM2 ni distiroidismo, $29 \%$ presentó hiperlipidemia y $30 \%$ cursó con hipertensión arterial sistémica controlada. En estos pacientes, la artritis reumatoide se encontró estable y en remisión (DAS28 de 2,4 \pm 0,1 y velocidad de sedimentación globular de 21,8 \pm $3,4 \mathrm{~mm} / \mathrm{h}$ ). Todos estaban siendo tratados a dosis estables con metotrexate y antiinflamatorios no esteroideos, mientras que 7 pacientes se encontraban recibiendo prednisona a dosis estable de 10 mg o menos por día. Ningún paciente fue tratado previamente con HCQ, ni estaban siendo tratados con otros fármacos antiartríticos, ni fármacos que modifiquen la SI o el metabolismo del colesterol. Las características demográficas, antropométricas y clínicas del grupo de estudio se muestran en la Tabla 1.

Las variables KITT, HDL-C, LDL-C, CT, TG, glucosa, insulina, ácido úrico, peso e IMC tuvieron

Tabla 1. Características demográficas, antropométricas y clínicas de la población de estudio antes de iniciar la intervención

\begin{tabular}{|lr|}
\hline Variable & \multicolumn{1}{c|}{ Valor* $^{*}$} \\
\hline Género (femenino/masculino) & $15 / 0$ \\
\hline Edad (años) & $46,9 \pm 11$ \\
\hline Evolución de la enfermedad (años) & $8,5 \pm 5,8$ \\
\hline Estatura (cm) & $156,5 \pm 16$ \\
\hline Peso (kg) & $75,8 \pm 9,1$ \\
\hline Índice de masa corporal $\left(\mathrm{kg} / \mathrm{m}^{2}\right)$ & $30,9 \pm 3,3$ \\
\hline Perímetro de la cintura $(\mathrm{cm})$ & $91,7 \pm 4,3$ \\
\hline Velocidad de sedimentación globular (mm/h) & $21,8 \pm 3,4$ \\
\hline DAS28 & $2,4 \pm 0,1$ \\
\hline Tensión arterial sistólica $(\mathrm{mmHg})$ & $123 \pm 15,2$ \\
\hline Tensión arterial diastólica $(\mathrm{mmHg})$ & $76 \pm 10,5$ \\
\hline
\end{tabular}

*promedio \pm desviación estándar. DAS28 = Índice de actividad de la enfermedad para 28 articulaciones. 
Tabla 2. Comparación entre las características antropométricas y bioquímicas, antes (basal) y después (final) de la intervención

\begin{tabular}{|c|c|c|c|}
\hline Variable & Basal* & Final* & $\mathbf{p}$ \\
\hline Peso $(k g)$ & $75,8 \pm 9,1$ & $74,7 \pm 10$ & 0,13 \\
\hline Índice de masa corporal (kg/m²) & $30,9 \pm 3,3$ & $30,4 \pm 3,5$ & 0,11 \\
\hline KITT (\%/min) & $4,27 \pm 1,2$ & $4,8 \pm 1,1$ & 0,03 \\
\hline $\mathrm{HDL}-\mathrm{C}(\mathrm{mg} / \mathrm{dL})$ & $46,7 \pm 15,7$ & $53,0 \pm 10,9$ & 0,27 \\
\hline LDL-C (mg/dL) & $119,7 \pm 48,7$ & $107,8 \pm 3$ & 0,25 \\
\hline VLDL-C (mg/dL) & $27,9 \pm 10,9$ & $25 \pm 8,1$ & 0,37 \\
\hline Colesterol total (mg/dL) & $204 \pm 41,8$ & $186,1 \pm 31,8$ & 0,04 \\
\hline Triglicéridos (mg/dL) & $153,5 \pm 59,9$ & $124,1 \pm 39,3$ & 0,01 \\
\hline Glucosa (mg/dL) & $83,6 \pm 18,2$ & $89 \pm 8,2$ & 0,05 \\
\hline Insulina $(\mu \mathrm{UI} / \mathrm{mL})$ & $19,6 \pm 9,2$ & $19,3 \pm 8,3$ & 0,85 \\
\hline Ácido úrico (mg/dL) & $4,53 \pm 0,88$ & $4,74 \pm 0,99$ & 0,14 \\
\hline
\end{tabular}

*promedio \pm desviación estándar. HDL-C = Colesterol de las lipoproteínas de alta densidad. LDL-C = Colesterol de las lipoproteínas de baja densidad. VLDL-C = Colesterol de las lipoproteínas de muy baja densidad. KITT = Tasa de depuración de glucosa sérica (sensibilidad a la insulina).

una distribución normal; a diferencia del VLDL-C. Para el análisis de las primeras se utilizó la prueba paramétrica tipo T de Student para grupos correlacionados, y para la última la prueba no paramétrica de Suma de Rangos de Wilcoxon.

Los valores de KITT, glucosa sérica y perfil de lípidos antes de iniciar la intervención farmacológica (basales) se muestran en la Tabla 2. Se observa que los pacientes presentaron una SI promedio de $4,27 \pm 1,2 \% /$ min y un perfil lipídico con CT y TG elevados, así como HDL-C por debajo de los valores recomendados. Los niveles de glucosa de ayuno se encontraron dentro del rango de referencia.

Al comparar las características antropométricas y metabólicas basales y finales, se encontró una diferencia estadísticamente significativa en los niveles de CT y TG. En cuanto a los niveles de HDL-C, LDL-C y VLDL-C se observó una tendencia a la mejoría aunque no se alcanzó una diferencia estadísticamente significativa. Los niveles de glucosa incrementaron al final del período de intervención en relación con los niveles basales, observándose una tendencia a la significancia estadística. No se encontraron diferencias significativas en las variables antropométricas al final del estudio.

Después de los tres meses de tratamiento con $400 \mathrm{mg}$ diarios de HCQ, la SI mejoró significati- vamente como se puede apreciar en la Tabla 2. El valor basal promedio de KITT fue de 4,27 $\pm 1,2 \%$ / min y el final de $4,80 \pm 1,1 \% / \mathrm{min}(\mathrm{p}=0,03)$.

El apego al tratamiento fue de $86,7 \%$ y se consideró satisfactorio. Sólo dos pacientes presentaron molestias gastrointestinales (meteorismo) transitorias de intensidad leve durante la primera semana de tratamiento que no obligaron a suspenderlo. No se presentaron eventos adversos serios.

\section{Discusión}

El presente trabajo es el primero en su género que evalúa y demuestra un efecto positivo de la HCQ sobre la SI en pacientes mexicanos con AR, tratados con $400 \mathrm{mg}$ diarios de HCQ vía oral durante 3 meses.

Algunos estudios clínicos reportan que la SI está disminuida cuando hay mayor actividad inflamatoria y que al mejorar ésta, también hay una recuperación de la $\mathrm{SI}^{26-29}$. Los pacientes que participaron en este trabajo se encontraban con AR clínicamente inactiva (DAS28 de 2,4 $\pm 0,1$ ) y con velocidad de sedimentación globular normal $(21,8 \pm 3,4 \mathrm{~mm} / \mathrm{h})$, lo que hace pensar que el efecto demostrado de la HCQ sobre la SI sea fármaco-específico. Con la finalidad de evitar el 
efecto Hawthorne, dado que no se incluyó un grupo control con placebo, se instruyó a los pacientes para que no modificaran su estilo de vida durante el tiempo en que estuvieron sometidos a la intervención con HCQ. A pesar de lo anterior, no podemos excluir completamente la posibilidad de que otros factores pudieron afectar la SI, sin embargo, el peso y el IMC permanecieron sin cambios al final del estudio, lo que apoya que la mejoría en la SI tuvo como causa más probable el tratamiento con HCQ. Debido a que existe una relación entre inflamación y disminución en la SI, es posible que la HCQ tenga un efecto benéfico más profundo en pacientes con AR activa que presenten una alteración de mayor magnitud en la $\mathrm{SI}^{30}$.

En el presente estudio se obtuvo un incremento en los niveles de glucosa sérica con tendencia a ser significativo al final de la intervención, aunque las cifras se mantuvieron dentro de los límites de referencia. Esto contrasta con algunos trabajos en los que se demostró que la HCQ disminuye los niveles plasmáticos de glucosa ${ }^{31,32}$. Si bien no tenemos una explicación satisfactoria para este resultado, dos reportes en la literatura, con diseño similar al presente, donde se valoró el efecto del tratamiento con infliximab sobre la SI en pacientes con AR, obtuvieron resultados similares; es decir, se observó un incremento en la SI pero los niveles de glucosa fueron mayores al final del tratamiento ${ }^{27,33}$. Parece ser que los mecanismos por los cuales la HCQ pudiera disminuir los niveles de glucosa son independientes de la mejoría en la SI y dependerían principalmente de interferir con el metabolismo de la insulina.

La HCQ también muestra efectos benéficos sobre el metabolismo de los lípidos en diversos estudios clínicos, a través de una disminución del CT y los TG séricos, y un incremento en los niveles sanguíneos de HDL-C $\mathrm{C}^{15,16,33-35}$, situación que no se demostró completamente en el presente estudio ya que sólo se encontró una diferencia significativa en los niveles séricos de CT y TG antes y después de la intervención, lo que no se pudo demostrar para HDL-C, LDL-Cy VLDL-C, aunque mostraron una tendencia hacia la mejoría al final del estudio. Cabe señalar que el no haber encontrado cambios significativos en los niveles de HDL-C, pudo deberse a un tamaño de muestra pequeño, a pesar de que éste fue calculado a partir de la fórmula para variables continuas en estudios antes-después, situación que debe tomarse como una limitación de este trabajo.
Diversos estudios epidemiológicos demuestran una asociación entre los niveles séricos de ácido úrico con el síndrome metabólico y sus componentes individuales, encontrando que niveles elevados de ácido úrico se relacionan con la hipertensión arterial, hiperinsulinemia, incremento en el IMC y el consumo de alcohol ${ }^{36-38}$. Sin embargo, no existe suficiente información sobre la relación existente entre los niveles de ácido úrico sérico y el síndrome metabólico, particularmente entre individuos con niveles normales de glucosa sérica. En el presente estudio no se encontró diferencia significativa entre las concentraciones séricas de ácido úrico e insulina antes y después del tratamiento con HCQ, lo que sugiere que el efecto de este fármaco sobre la SI es a nivel postreceptor.

$\mathrm{Si}$ bien no se conoce un valor de referencia para KITT, en dos estudios clínicos realizados en población mexicana sana y con un IMC $<25 \mathrm{~kg} /$ $\mathrm{m}^{2}$ los valores de KITT se encontraron entre 4,5y $4,9 \% / \mathrm{min}^{39,40}$. Como no se tienen reportes previos en los que se haya utilizado la PTI en pacientes con AR, ni se realizó en el presente estudio una prueba piloto para establecer un valor de KITT en pacientes con AR inactiva que permitiera un cálculo más adecuado del tamaño de la muestra, se tomó como referencia un valor de desviación estándar de KITT de 0,8\%/min a partir de un estudio con diseño similar al presente ${ }^{25}$. A pesar de esto, el tamaño de muestra utilizado en esta investigación fue suficiente para encontrar diferencia significativa en la SI $(\mathrm{p}=0,03)$.

Finalmente, la HCQ en general fue bien tolerada en la población de estudio y no se reportaron eventos adversos serios. El porcentaje de síntomas gastrointestinales fue menor a $15 \%$ y todos transitorios, lo cual se encuentra acorde con lo reportado en la literatura ${ }^{8}$. Otro de los efectos desfavorables que puede presentarse por el uso de antimaláricos en pacientes con AR es la retinopatía, la cual se ha reportado hasta en $10 \%$ con el uso de cloroquina y aproximadamente $1 \%$ con el uso prolongado (más de cinco años) de $\mathrm{HCQ}^{10}$. En lo que respecta a la utilización de este último fármaco se recomienda una dosis de 200 a $400 \mathrm{mg}$ diarios $(\leq 6 \mathrm{mg} / \mathrm{kg} /$ día). No hay casos reportados de retinopatía por HCQ con la dosis y frecuencia utilizadas en esta investigación.

En conclusión, la administración de $400 \mathrm{mg}$ diarios de HCQ vía oral durante tres meses, mejoró significativamente la SI en pacientes con 
AR inactiva, así como los niveles séricos de CT y TG. Esto abre la posibilidad de iniciar estudios a mediano y largo plazo para evaluar otras variables que influyen en la SI y el desarrollo de aterosclerosis temprana, así como el efecto de este fármaco en la prevención y tratamiento de patologías no reumáticas que cursan con disminución de la SI.

\section{Referencias}

1. McCarty DJ. Clinical picture of rheumatoid arthritis. In: McCarty DJ, Editor, Arthritis and Allied Conditions: A Textbook of Rheumatology. Philadelphia, USA: Lea \& Febiger 1989; p. 605-19.

2. Gazi IF, Boumpas DT, Mikhailidis DP, Ganotakis ES. Clustering of cardiovascular risk factors in rheumatoid arthritis: the rationale for using statins. Clin Exp Rheumatol 2007; 25 (1): 102-11.

3. del Rincón ID, Williams K, Stern MP, Freeman GL, Escalante A. High incidence of cardiovascular events in a rheumatoid arthritis cohort not explained by traditional cardiac risk factors. Arthritis Rheum 2001; 44 (12): 2737-45.

4. La Montagna G, Cacciapuoti F, Buono R, Manzella D, Mennillo GA, Arciello A, et al. Insulin resistance is an independent risk factor for atherosclerosis in rheumatoid arthritis. Diab Vasc Dis Res 2007; 4 (2): 130-5.

5. Dessein PH, Tobias M, Veller MG. Metabolic syndrome and subclinical atherosclerosis in rheumatoid arthritis. J Rheumatol 2006; 33 (12): 2425-32.

6. Dessein PH, Joffe BI. Insulin resistance and impaired beta cell function in rheumatoid arthritis. Arthritis Rheum 2006; 54 (9): 2765-75.

7. Ferrannini E, Mari A. How to measure insulin sensitivity. J Hypertens 1998; 16 (7): 895-906.

8. Restrepo JF. Antimaláricos. En Alarcón-Segovia D, Molina J, Editores, Tratado Hispanoamericano de Reumatología. Bogotá, Colombia: Schering Plough; 2006. p. 311-20.

9. Tutor-Ureta P, Yebra-Bango M. Los antimaláricos en las enfermedades sistémicas. Rev Clin Esp 2005; 205 (5): 230-2.

10. Wallace DJ. Antimalarial Therapies. In: Wallace DJ \& Hahn BH, Editors, Dubois' Lupus Erythematosus. Philadelphia, USA: Lippincott Williams \& Wilkins; 2007. p. 1152-76.

11. Emami J, Pasutto FM, Mercer JR, Jamali F. Inhibition of insulin metabolism by hydroxychloroquine and its enantiomers in cytosolic fraction of liver homogenates from healthy and diabetic rats. Life Sci 1999; 64 (5): 325-35.
12. Emami J, Gerstein HC, Pasutto FM, Jamali F. Insulinsparing effect of hydroxychloroquine in diabetic rats is concentration dependent. Can J Physiol Pharmacol 1999; 77 (2): 118-23.

13. Gerstein HC, Thorpe KE, Taylor DW, Haynes RB. The effectiveness of hydroxychloroquine in patients with type 2 diabetes mellitus who are refractory to sulfonylureas-a randomized trial. Diabetes Res Clin Pract 2002; 55 (3): 209-19.

14. Wasko MC, Hubert HB, Lingala VB, Elliott JR, Luggen ME, Fries JF, et al. Hydroxychloroquine and risk of diabetes in patients with rheumatoid arthritis. JAMA 2007; 298 (2): 187-93.

15. Sabio JM, Zamora-Pasadas M, Jiménez-Jáimez J, Albadalejo F, Vargas-Hitos J, Rodríguez del Águila MD, et al. Metabolic syndrome in patients with systemic lupus erythematosus from Southern Spain. Lupus 2008; 17 (9): 849-59.

16. Petri M. Hydroxychloroquine use in the Baltimore Lupus Cohort: effects on lipids, glucose and thrombosis. Lupus 1996; 5 Suppl 1: S16-22.

17. Arnett FC, Edworthy SM, Bloch DA, McShane DJ, Fries JF, Cooper NS, et al. The American Rheumatism Association 1987 revised criteria for the classification of rheumatoid arthritis. Arthritis Rheum 1988; 31 (3): 315-24.

18. Fransen J, Creemers MC, Van Riel PL. Remission in rheumatoid arthritis: agreement of the disease activity score (DAS28) with the ARA preliminary remission criteria. Rheumatology 2004; 43 (10): 1252-5.

19. Grundy SM, Cleeman JI, Daniels SR, Donato KA, Eckel RH, Franklin BA, et al. Diagnosis and management of the metabolic syndrome: an American Heart Association/National Heart, Lung, and Blood Institute Scientific Statement. Circulation 2005; 112 (17): 2735-52.

20. American Diabetes Association. Diagnosis and classification of diabetes mellitus. Diabetes Care 2008; 31 Suppl 1: S55-60.

21. Aguilar-Salinas CA. Fisiopatología de la diabetes mellitus tipo 2 y de la resistencia a la insulina. México, D.F.: Intersistemas, S.A. de C.V.; 2008. p. 41-89.

22. Bonora E, Moghetti P, Zancanaro C, Cigolini M, Querena $\mathrm{M}$, Cacciatori $\mathrm{V}$, et al. Estimates of in vivo insulin action in man: comparison of insulin tolerance test with euglycemic and hyperglycemic glucose clamp studies. J Clin Endocrinol Metab 1989; 68 (2): 374-8.

23. González-Ortiz LJ, Martínez-Abundis E, González-Ortiz $\mathrm{M}$. A new model to fit glucose concentration during the insulin tolerance test improving the predictive capability to estimate insulin sensitivity. Nutr Metab Cardiovasc Dis 2006; 16 (1): 78-9. 
24. Pagano M, Kimberlee G. Cálculo del tamaño de muestreo. En: Fundamentos de Bioestadística. México, D.F.: International Thomson Editores S.A.; 2001: p. 330-41.

25. Grover-Páez F, Martínez Abundis E, González Ortiz M. [Effect of hormone replacement therapy including a progestin on insulin sensitivity and lipid profile in postmenopausal women]. Rev Med Chile 2001; 129 (9): 989-94.

26. Tam LS, Tomlinson B, Chu TT, Li TK, Li EK. Impact of TNF inhibition on insulin resistance and lipids levels in patients with rehumatoid arthritis. Clin Rheumatol 2007; 26 (89): 1799-800

27. Oguz FM, Oguz A, Uzunlulu M. The effect of infliximab treatment on insulin resistance in patients with rheumatoid arthritis. Acta Clin Belg 2007; 62 (4): 218-22.

28. Dessein PH, Joffe BI, Stanwix AE, Christian BF, Veller M. Glucocorticoids and insulin sensitivity in rheumatoid arthritis. J Rheumatol 2004; 31 (5): 867-74.

29. Hällgren R, Berne C. Glucose intolerance in patients with chronic inflammatory diseases is normalized by glucocorticoids. Acta Med Scand 1983; 213 (5): 351-5.

30. Dessein PH, Joffe BI, Stanwix AE. Editorial: should we evaluate insulin sensitivity in rheumatoid arthritis? Semin Arthritis Rheum 2005; 35 (1): 5-7.

31. Petri M, Ferman D, Goldman D. Hypoglycemic effect of hydroxychloroquine in systemic lupus erythematosus [abstract]. Arthritis Rheum 1994; 37 (Suppl): R24.

32. Penn SK, Kao AH, Schott LL, Elliott JR, Toledo FG, Kuller L, et al. Hydroxychloroquine and glycemia in women with rheumatoid arthritis and systemic lupus erythematosus. J Rheumatol 2010; 37 (6): 1136-42.

33. González-Gay MA, De Matias JM, González-Juataney C, García Porrua C, Sánchez-Andrade A, Martin J, et al.
Anti-tumor necrosis factor- $\alpha$ blockade improves insulin resistance in patients with rheumatoid arthritis. Clin Exp Rheumatol 2006; 24 (1): 83-6.

34. Wallace DJ, Metzger AL, Stecher VJ, Turnbull BA, Kern PA. Cholesterol-lowering effect of hydroxychloroquine in patients with rheumatic disease: reversal of deleterious effects of steroids on lipids. Am J Med 1990; 89 (3): 322-6.

35. Munro R, Morrison E, McDonald AG, Hunter JA, Madhok R, Capell HA. Effect of disease modifying agents on the lipid profiles of patients with rheumatoid arthritis. Ann Rheum Dis 1997; 56 (6): 374-7.

36. Tsouli SG, Liberopoulos EN, Mikhailidis DP, Athyros VG, Elisaf MS. Elevated serum uric acid levels in metabolic syndrome: an active component or an innocent bystander? Metabolism 2006; 55 (10): 1293-301.

37. Yoo TW, Sung KC, Shin HS, Kim BJ, Kim BS, Kang JH, et al. Relationship between serum uric acid concentration and insulin resistance and metabolic syndrome. Circ J 2005; 69 (8): 928-33.

38. Lin SD, Tsai DH, Hsu SR. Association between serum uric acid level and components of the metabolic syndrome. J Chin Med Assoc 2006; 69 (11): 512-6.

39. González-Ortíz M, Martínez-Abundis E, CardonaMuñoz EG, Lifshitz A, Quiñones-Galván A. Metabolic profile and insulin sensitivity in healthy young Mexicans with a strong family history of non-insulin-dependent diabetes mellitus in the paternal branch. Arch Med Res 1997; 28 (3): 421-4.

40. González-Ortiz M, Martínez-Abundis E. Maternal effect of Type 2 diabetes mellitus on insulin sensitivity and metabolic profile in healthy young Mexicans. Diabetes Nutr Metab 1999; 12 (1): 32-6. 